\title{
Conjunctival-corneal melt in association with carotid artery stenosis
}

\author{
Rosalind MK Stewart' \\ Say Aun Quah' \\ Dan Q Nguyen ${ }^{2}$ \\ Stephen B Kaye' \\ 'Royal Liverpool University Hospital, \\ Liverpool, UK; ${ }^{2}$ Bristol Eye Hospital, \\ Bristol, UK
}

Purpose: To report a case of severe conjunctival-corneal melt in association with carotid artery stenosis.

Methods: Observational case report.

Results: A 76-year-old man with a history of bilateral severe carotid artery occlusion and nonarteritic ischemic optic neuropathy developed a spontaneous bulbar conjunctival defect. Despite intensive lubrication, and attempts at surgical closure including an amniotic membrane patch graft, it progressed with subsequent adjacent corneal perforation. Thorough investigations revealed no underlying disease, except markedly delayed episcleral vessel filling on anterior segment fluorescein angiography.

Conclusions: Neovascularisation is a known factor in the inhibition of ulceration. In light of the findings in this report, ocular ischemia should be considered as a cause or contributing factor in the differential diagnosis of conjunctival-corneal melt.

Keywords: conjunctival melt, corneal melt, ocular ischemia, carotid artery stenosis

\section{Introduction}

Corneo-scleral melt is a severe ocular condition characterized by progressive tissue thinning often leading to perforation. It may be initiated by a wide variety of underlying factors, most commonly systemic or local autoimmune disease, microbial infection or previous surgery. Anterior segment ischemia following recti muscle surgery and alkali burns has also been implicated. Carotid artery stenosis may cause ocular ischemia either of embolic nature or due to chronic hypoperfusion. One case of ischemic scleral melt due to carotid artery obstruction has been reported (Schotveld et al 2006), but to our knowledge no such case of conjunctival-corneal melt has been previously described.

\section{Case report}

A 76-year-old man with a history of left nonarteritic ischemic optic neuropathy, presented with irritation in the same eye. A perilimbal area of conjunctival thinning with surrounding injection and central epithelial defect was noted temporally. The eye was otherwise quiet with no corneal edema, anterior chamber activity or clinically apparent iris atrophy. Microbiology and virology testing were negative. He did not exhibit any clinical features of systemic vasculitis (including giant cell arteritis) or autoimmune disease. Full blood count and inflammatory markers were within normal ranges (ESR 16 , CRP $<5$ ). Autoimmune screen (RF, nuclear mitochondrial and smooth muscle ANA, pANCA, cANCA, dsDNA screen, ENA screen, C3 and 4 complement, and cryoglobulins) was negative. Despite the use of intensive antibiotics, bandage contact lenses (22/9.2 mm plano, $72 \%$ water content), punctal plugs, lubricants and topical steroids, the defect gradually increased in size (Figure 1). All investigations were repeated and normal. Multiple attempts to surgically close the defect led only to rapid 


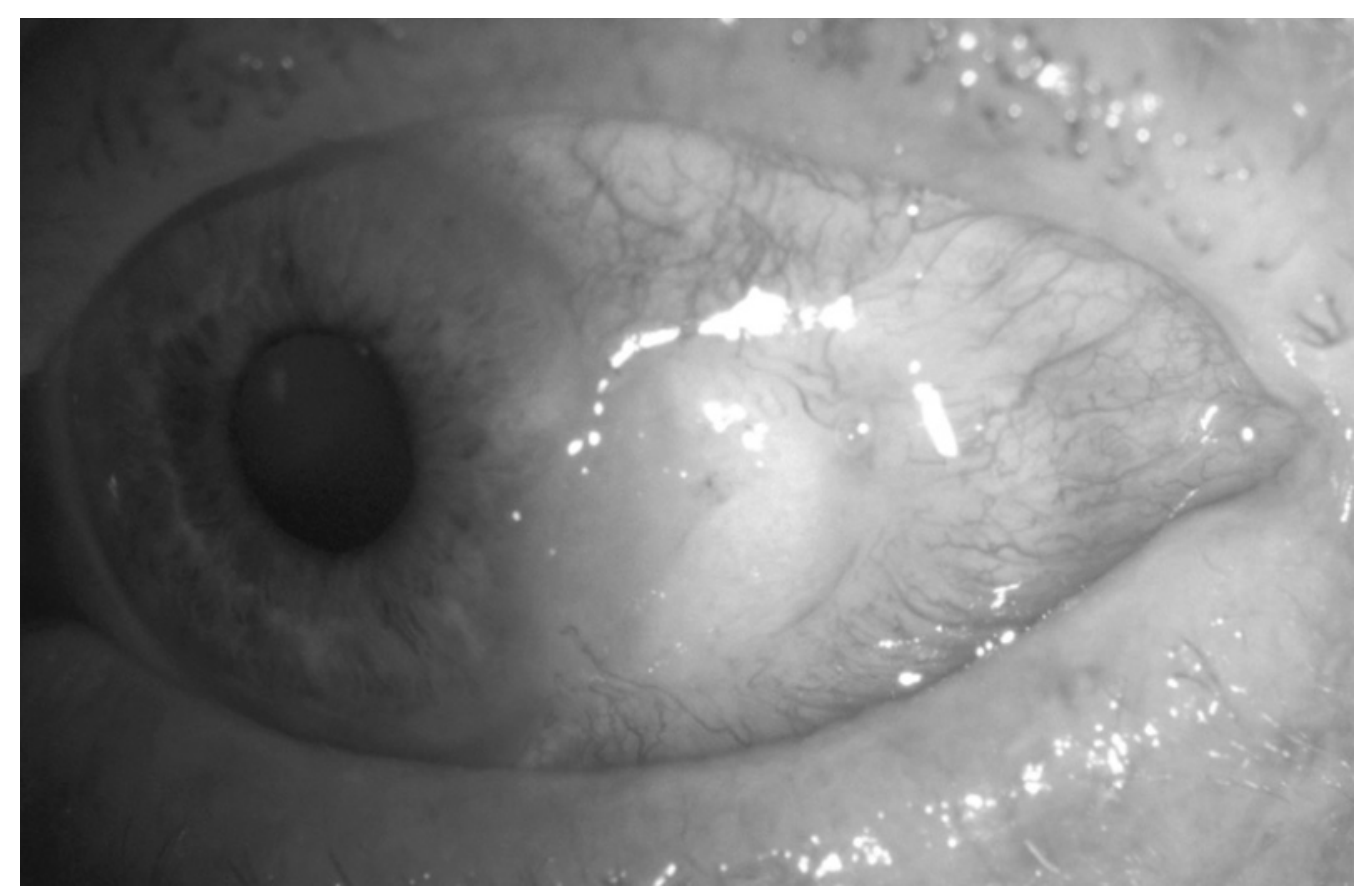

Figure I Spontaneous nonhealing conjunctival defect.

recurrence, and an amniotic membrane patch graft to aid conjunctival healing dissolved within two days. Biopsies on three separate occasions showed moderate chronic inflammatory cell infiltrate with no evidence of neoplasia. Despite regular follow-up and intensive lubrication he developed adjacent corneal thinning and subsequent perforation. This was successfully glued and the eye settled somewhat albeit with a large persistent conjunctival defect (Figure 2).

Our patient was known to have severe carotid artery stenosis (right $>90 \%$, left totally occluded) causing

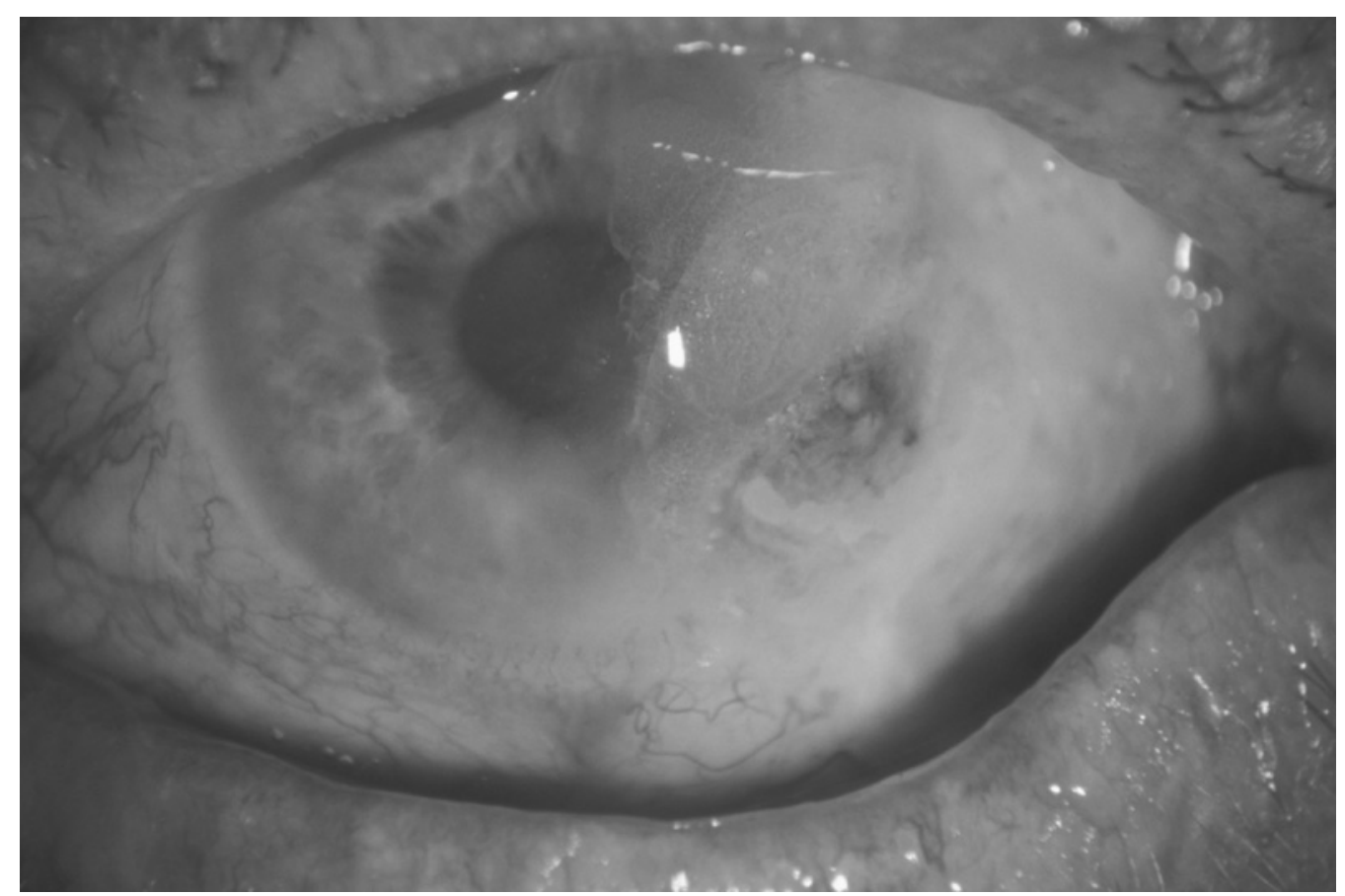

Figure 2 Large persistent conjunctival defect post corneal gluing. 
symptomatic transient ischemic attacks, but endarterectomy was declined due to significant anesthetic risks. Doppler scanning confirmed reduced flow bilaterally in the common carotid arteries, absent flow of the left internal carotid artery, a tight stenosis with peak systolic velocity of $400 \mathrm{~cm} / \mathrm{second}$ in the right internal carotid artery, and reversed flow in the left supratrochlear artery. Anterior segment fluorescein angiography showed marked delay in antecubital-episcleral perfusion times to both eyes: right 38 , left (affected eye) 52 seconds. He had widespread severe cardiovascular disease, suffered increasing transient ischemic attacks and unfortunately died soon afterwards from myocardial infarction.

\section{Discussion}

Anterior segment ischemia may cause corneoscleral melt, being well documented following chemical injuries and recti surgery. It has also been incidentally described in conjunction with eyelid necrosis following retrobulbar anesthesia (3 $\mathrm{ml}$ of lidocaine $2 \%$ with epinephrine [1:10,000]) (Sharma et al 2003). Schotveld and colleagues (2006) recently reported the case of a 71 year-old man with progressive scleral melting and two failed conjunctival grafts, in which the only risk factor found was significant carotid stenosis. Normal carotid Doppler flow was demonstrated following endarterectomy, after which the ocular situation stabilized.

This case was extensively negatively-investigated, and melting progressed despite intensive lubrication, antibiotics, and surgical intervention. He had an extensive history of vascular disease with severe carotid artery stenosis; the left, ipsilateral to both the nonarteritic ischemic optic neuropathy and conjunctival-corneal melt, being totally occluded. Antecubital-episcleral perfusion times were markedly delayed on fluorescein angiography (normal 8-23 seconds depending on height [Ormerod et al 1995]), again, more so to the left. In the absence of other risk factors for conjunctivalcorneal melt this cannot be ignored.

Occlusive carotid artery disease may produce acute ophthalmic manifestations due to embolism, or chronic manifestations associated with progressive ocular ischemia. Chronic ischemia produces ocular ischemic syndrome, characterised by venous dilatation, midperipheral intraretinal hemorrhages, macular edema, neovascularisation, cataract, and aqueous flare. Although our case demonstrated no features of classic ocular ischemic syndrome, these findings are by no means universal with severe carotid stenosis (Costa et al 1997). There was however evidence of nonarteritic anterior ischemic optic neuropathy in the same eye. The latter appears to result from perfusion insufficiency in the short posterior ciliary arteries leading to infarction of the retrolaminar portion of the optic disc (Matthews 2005), and has been associated with ocular ischemic syndrome as a complication of carotid artery occlusion (Brown 1986). Similarly chronic hypoperfusion of the anterior ciliary/episcleral arteries may result in localized/focal anterior segment ischemia and subsequent melting.

Neovascularisation is an inhibitor of corneal ulceration (Conn et al 1980) possibly through the provision of inhibitors of the matrix metalloproteinases (MMPs) (Fini et al 1998). It is possible therefore, that in this case, the ischemia led to a relative deficiency of MMP inhibitors, with subsequent destruction of the reparative process. In addition, stimulated phagocytic cells not only produce active oxygen species during the respiratory burst, but also release neutral collagenase and acid lysosomal hydrolases that contribute to and amplify the degradation of the extracellular matrix (Carubelli et al 1990). It has been shown in skin that ischemia and hypoxia result in elevated collagen remodeling in an oxygen-poor environment, and that unstable collagen molecules are synthesized together with upregulated MMPs, resulting in collagen denaturation, defective angiogenesis, weaker skin, and predisposition to ulceration (Dalton et al 2007). A similar mechanism may therefore occur in the conjunctiva. In a rabbit model, oxygen therapy has been shown to reduce corneal ulceration (Sharifipour et al 2007). Ocular ischemia should therefore be considered as a causative or contributing factor to melting and ulceration of the ocular surface.

\section{Disclosure}

The authors have no conflicts of interest to report.

\section{References}

Brown GC. 1986. Anterior ischemic optic neuropathy occurring in association with carotid artery obstruction. J Clin Neuroophthalmol, 6:29-42.

Carubelli R, Nordquist RE, Rowsey JJ. 1990. Role of active oxygen species in corneal ulceration. Effect of hydrogen peroxide generated in situ. Cornea, 9:161-9.

Conn H, Berman M, Kenyon K, et al. 1980. Stromal vascularization prevents corneal ulceration. Invest Ophthalmol Vis Sci, 19:362-70.

Costa VP, Kuzniec S, Molnar LJ, et al. 1997. Clinical findings and hemodynamic changes associated with severe occlusive carotid artery disease. Ophthalmology, 104:1994-2002.

Dalton SJ, Whiting CV, Bailey JR, et al. 2007. Mechanisms of chronic skin ulceration linking lactate, transforming growth factor-beta, vascular endothelial growth factor, collagen remodeling, collagen stability, and defective angiogenesis. J Invest Dermatol, 127:958-68.

Fini ME, Cook JR, Mohan R. 1998. Proteolytic mechanisms in corneal ulceration and repair. Arch Dermatol Res, 290(Suppl):S12-23. 
Mathews MK. 2005. Nonarteritic ischemic optic neuropathy. Curr Opin Ophthalmol, 16:341-5.

Ormerod LD, Fariza E, Webb RH. 1995. Dynamics of external ocular blood flow studied by scanning angiographic microscopy. Eye, 9:605-14.

Schotveld JH, Beerthuizen JJG, Zaal MJW. 2006. Scleral melting in a patient with carotid artery obstruction. Cornea, 25:101-3.
Sharifipour F, Zamani M, Idani E, et al. 2007. Oxygen therapy for severe corneal alkali burn in rabbits. Cornea, 9:1107-10.

Sharma A, Gupta A, Bandyopadhyay S, et al. 2003. Necrosis of the eyelids and sclera after retrobulbar anaesthesia. J Cataract Refract Surg, 29:842-4. 